\title{
Mise en charge immédiate de mini-implants : à propos de deux cas cliniques
}

\section{Immediate loading of mini-implants: a clinical report of two cases}

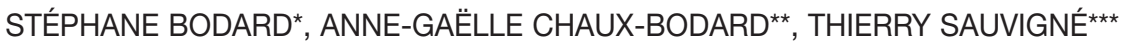

\section{RÉSUMÉ}

La mise en charge immédiate des mini-implants est en plein développement. Dans ces deux cas cliniques, leur utilisation a apporté aux patients une amélioration immédiate et durable de leur fonction masticatoire et de la stabilité prothétique. Ils ont réduit de manière considérable le traumatisme per- et post-opératoire, la durée globale et le coût du traitement. Dans le premier cas, ils permettent d'éviter la réalisation d'une greffe d'apposition osseuse en épaisseur; dans le second cas, de stabiliser de manière simple et rapide une prothèse complète mandibulaire. Ces cas cliniques sont un premier exemple de l'utilisation des miniimplants à moyen terme en posant les bases d'une nouvelle alternative à l'implantologie conventionnelle. (Med Buccale Chir Buccale 2003; 9: 29-36).

mots clés : Implant dentaire, Mise en charge immédiate, Prothèse mandibulaire

médecine buccale chirurgie buccale

VOL. $9, \mathrm{~N}^{\circ} 1$ 2003

page 29

\section{SUMMARY}

Immediate loading of dental mini-implants is in full development. In these two case reports, their use brings to patients an immediate and durable increase in swelling and in denture stability. They decrease per- and postoperative traumatism, global delay and treatment cost. In the first case, they avoid a bone graft; in the second case, they allow mandibular denture stabilisation in a fast and simple way. These case reports are a first example of middle-term mini-implant use and build bases of a new alternative in conventional implantology. (Med Buccale Chir Buccale 2003; 9: 29-36).

key words : Dental implant, Immediate loading, Mandibular denture

\footnotetext{
* Hôpital St Roch, Nice, France

** Centre Léon Bérard, Lyon, France

*** Hôpital de l'Antiquaille, Lyon, France
}

Demande de tirés à part :

Stéphane Bodard 30, grande rue de la Guillotière 69007 LYON

Article reçu le 28 mars 2002. Accepté pour publication le 5 juillet 2002. 
médecine

buccale

chirurgie

buccale

VOL. $9, \mathrm{~N}^{\circ} 1$ 2003

page 30
Les bases de l'implantologie actuelle ont été posées dès les années 1970 par Bränemark et coll. [1] en définissant le concept d' "ostéointégration". Les techniques et les systèmes implantaires ont beaucoup évolué depuis cette date [2] ; d'une part vers une réduction de la durée globale du traitement et d'autre part vers une simplification des protocoles. Actuellement, de nombreuses études portent sur des délais de mise en charge raccourcis voire même une mise en charge immédiate [3-8]. Ces études ont un taux de succès variable selon les auteurs, mais ce taux bien que satisfaisant reste légèrement inférieur à celui obtenu avec une mise en charge différée.

Parallèlement, des systèmes de mini-implants transitoires ou provisoires ont été développés par différentes sociétés ; ils sont mis en charge immédiatement pour maintenir une prothèse adjointe [9] ou conjointe [10] pendant la période d'intégration des implants conventionnels [11]. Le but de l'exposé est de proposer d'autres indications pour la pose de mini-implants à travers la présentation de deux cas cliniques.

\section{OBSERVATION}

\section{Matériel}

Certains auteurs comme Vigolo [12] désignent par le terme de «mini-implant » des implants de diamètre légèrement réduit $(2,9 \mathrm{~mm})$. Les miniimplants Sendax MDI sont des implants endo-osseux auto-forants en alliage de titane TiAl6V4 de 1,8 mm de diamètre avec des longueurs disponibles de $10 \mathrm{~mm}, 13 \mathrm{~mm}, 15 \mathrm{~mm}$ et $18 \mathrm{~mm}$. Ce sont des implants monoblocs avec attachement sphérique intégré.

\section{Méthodes}

Après désinfection du champ cutanéo-muqueux, on procède à l'anesthésie locale au niveau des sites concernés. Les mini-implants sont mis en place directement au travers de la fibromuqueuse après forage. Cependant, en fonction de la largeur de la crête, on peut procéder à une incision crestale à l'aide d'une lame $n^{\circ} 15$, puis à un léger décollement de pleine épaisseur des lambeaux vestibulaire et lingual. Dans ce cas, les lambeaux sont suturés après la mise en place des implants. Le forage de la loge implantaire est effectué sous irrigation externe avec le foret pilote de $1,1 \mathrm{~mm}$ de diamètre monté sur contre-angle. L'implant est inséré dans la loge implantaire par vissage manuel à l'aide de différentes clés jusqu'au positionnement final. Une orthopantomographie de contrôle est réalisée à la fin de l'intervention.

\section{Cas clinique $n^{\circ} 1$}

Un homme âgé de 53 ans vient consulter le 6 septembre 2000. II présente un déficit fonctionnel notamment lors de la mastication et souhaiterait avoir une meilleure stabilité de sa prothèse adjointe mandibulaire. Le patient fume un paquet de cigarettes par jour, ce qui est à la limite de l'indication pour la pose d'implants. L'examen clinique révèle l'absence de nombreuses dents aussi bien au maxillaire (il persiste 13 et 26) qu'à la mandibule (il persiste 32, 33 ainsi que la 34 cariée à l'état de racine). La crête mandibulaire paraît avoir une bonne hauteur malgré une résorption qui augmente en direction distale ; en revanche, elle est d'une finesse extrême sur une grande partie de sa hauteur. L'orthopantomographie confirme la présence d'une hauteur d'os suffisante pour la pose des mini-implants et permet de préciser les rapports avec les structures anatomiques voisines. Le patient n'étant pas suffisamment motivé pour bénéficier d'une greffe osseuse afin d'augmenter la crête en épaisseur, il lui est alors proposé de mettre en place trois miniimplants. La 34 est extraite trois semaines avant la pose des implants ; c'est le temps nécessaire pour avoir une fermeture muqueuse au niveau du site extractionnel. Le matin de l'intervention, le patient est mis sous traitement de spiramycine + métronidazole (3 cp.j-1) pendant six jours, de méthylprednisolone 16 mg (3 cp.j-1) pendant trois jours, du paracétamol 1000 mg et de l'hexétidine. Compte tenu de la faible épaisseur de la crête alvéolaire, on réalise une incision et un décollement mucopériosté (Fig. 1). Une résection osseuse de l'ordre de $2 / 3 \mathrm{~mm}$ est réalisée au niveau des sites implantaires à l'aide d'une pincegouge afin d'obtenir une largueur de crête suffisante pour accueillir les implants de 1,8 mm de 

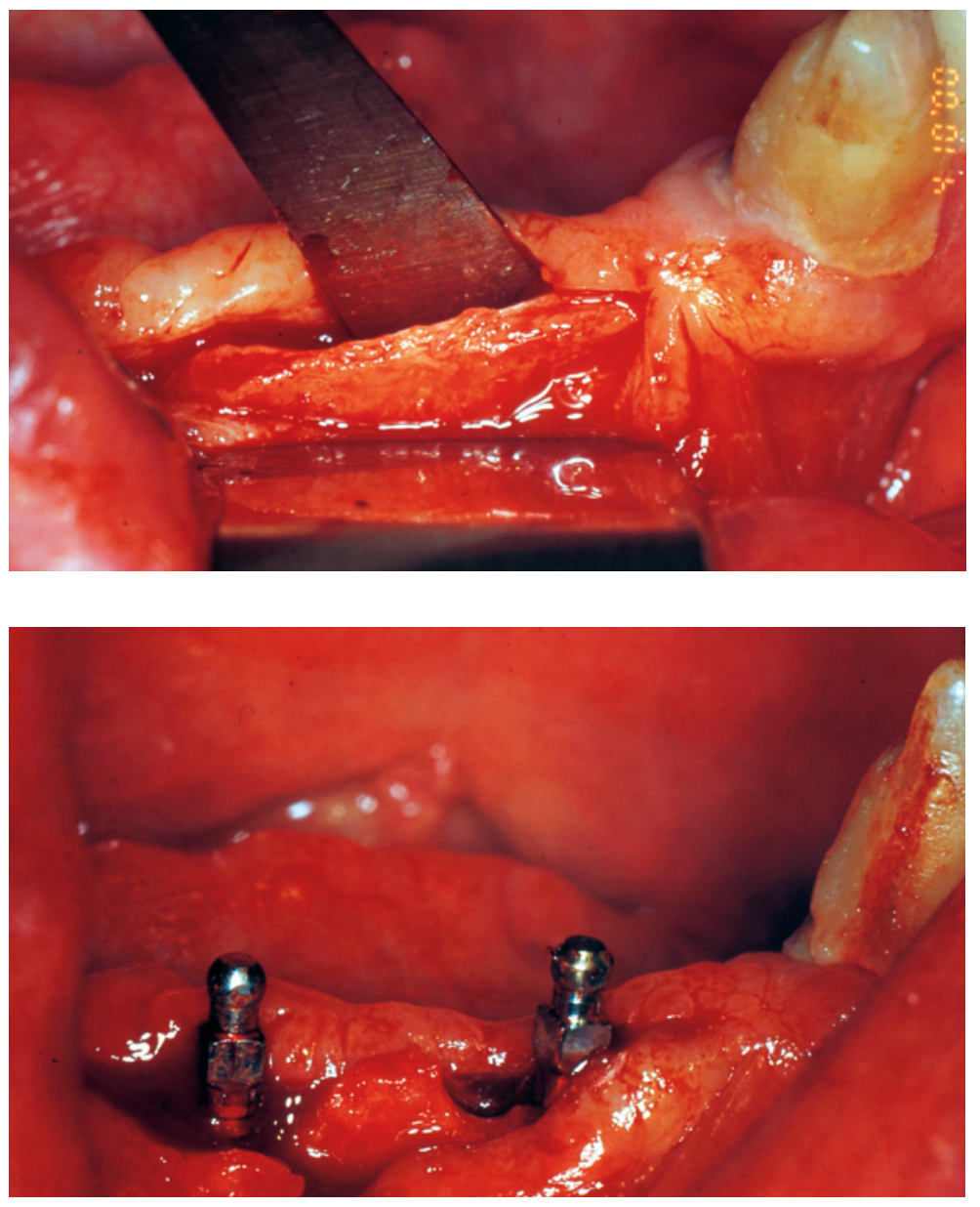

2

Figure 1 : Crête osseuse après décollement. Notez la finesse extrême de celle-ci.

Bone crest after flap detachment. Notice the extreme thinness.

Figure 2 : Mini-implants en place. Notez la résection osseuse réalisée à la pince gouge.

Mini implants in place. Notice the bone resection realised with gouge pliers

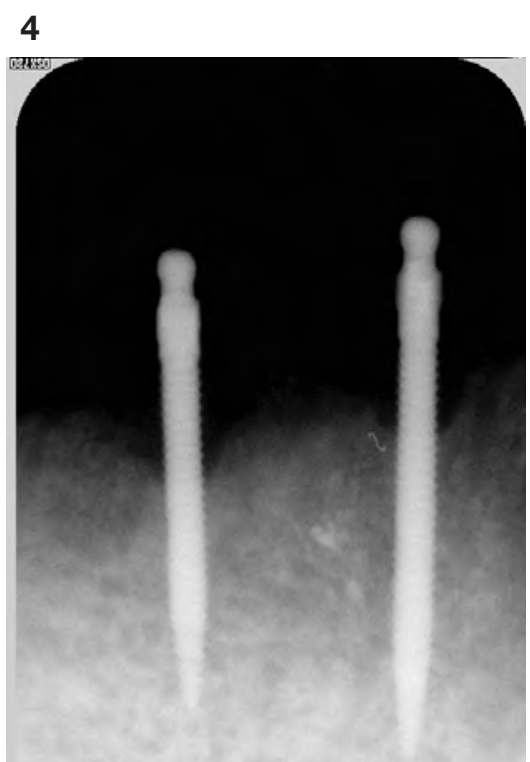

3

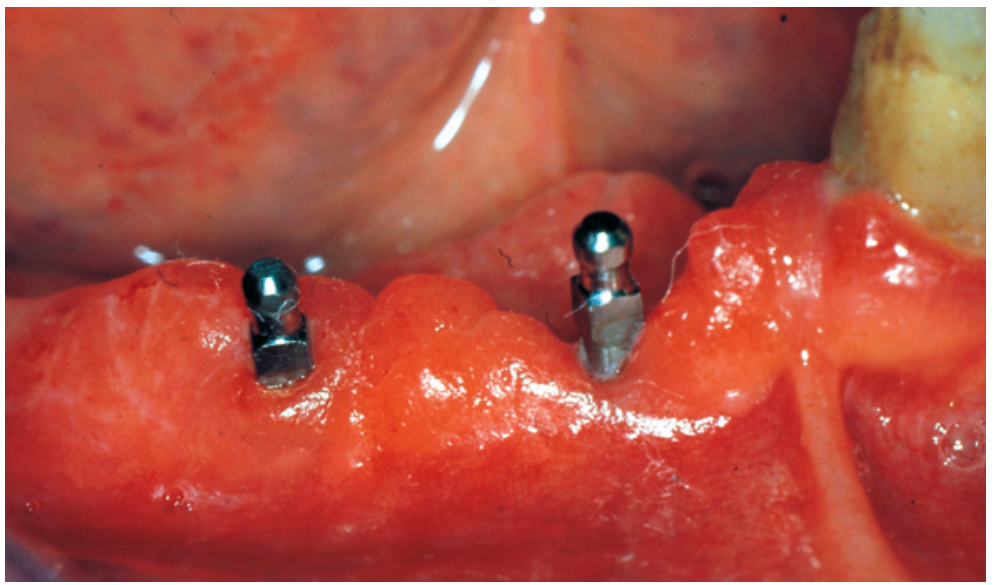

Figure 3 : Vue clinique 1 an après la mise en charge immédiate des mini-implants.

Clinical view 1 year after immediate loading of mini implants.

Figure 4 : : Radiographie rétroalvéolaire 1 an après la pose. La résection crestale est encore visible.

Radiograph 1 year after placement. Crestal resection is still visible. 
médecine

buccale

chirurgie

buccale

VOL. $9, \mathrm{~N}^{\circ} 1$ 2003

page 32 diamètre. La mise en place des implants est effectuée (Fig. 2), à savoir : un mini-implant de $18 \mathrm{~mm}$ en position 41, un mini-implant de $15 \mathrm{~mm}$ en position 43, un mini-implant de $15 \mathrm{~mm}$ en position 34.

Les lambeaux sont repositionnés et suturés à l'aide d'un fil de soie décimale 2 afin d'obtenir une coaptation parfaite des berges. La stabilité primaire de l'implant en position de 34 n'était pas suffisante pour permettre une mise en charge immédiate de celui-ci (site d'extraction récent). En revanche, la mise en place des deux attachements en 41 et 43 est réalisée dans la séance par adjonction de résine autopolymérisable.

Le patient est revu pour l'ablation des sutures la semaine suivante. II est à présent satisfait de la stabilité de sa prothèse mandibulaire et retrouve une fonction masticatoire correcte. L'attachement femelle en position de 34 est mis en place un mois après l'intervention, le mini-implant présente alors une excellente stabilité. Treize mois plus tard, les mini-implants donnent entière satisfaction, ne présentent aucun signe de mobilité, émettent un son clair à la percussion et sont parfaitement ostéointégrés radiographiquement (Fig. 3 et 4).

\section{Cas clinique $n^{\circ} 2$}

Un homme âgé de 80 ans vient consulter le 7 novembre 2000. Ses anciennes prothèses adjointes complètes maxillaire et mandibulaire viennent d'être refaites, mais sa prothèse mandi- bulaire reste instable lors de la mastication malgré la présence d'un attachement sur overdenture au niveau de la 33, seule dent résiduelle sur l'arcade. Le patient ne présente pas de contre-indications implantaires générales. A l'examen clinique, la crête mandibulaire paraît avoir un volume satisfaisant aussi bien en hauteur qu'en épaisseur. L'orthopantomographie confirme la présence d'un volume osseux suffisant pour la pose des implants et permet de préciser les rapports avec les structures anatomiques voisines. Le patient ne souhaite pas d'intervention longue dont les suites pourraient être douloureuses. De plus, le patient ne peut financer un traitement implantaire classique ; il lui est alors proposé de mettre en place quatre mini-implants et de procéder à l'extraction de la 33 résiduelle qui ne présente plus aucun rôle rétentif ni aucun intérêt prothétique. Le matin de l'intervention, le patient est mis sous traitement de clindamycine $300 \mathrm{mg}$ (3 cp.j-1) pendant six jours, du méthylprednisolone $16 \mathrm{mg}(3 \mathrm{cp} . \mathrm{j}-1)$ pendant trois jours et du paracétamol 1000 mg. Durant I'intervention, la canine mandibulaire est extraite de manière conventionnelle. On ne réalise aucune incision ni décollement ; on procède au forage de la loge implantaire directement à travers la fibromuqueuse. La mise en place des implants est alors effectuée manuellement (Fig. 5), à savoir : un mini-implant de $15 \mathrm{~mm}$ en position 34, un miniimplant de $18 \mathrm{~mm}$ en position 31, un mini-implant de $18 \mathrm{~mm}$ en position 41 .

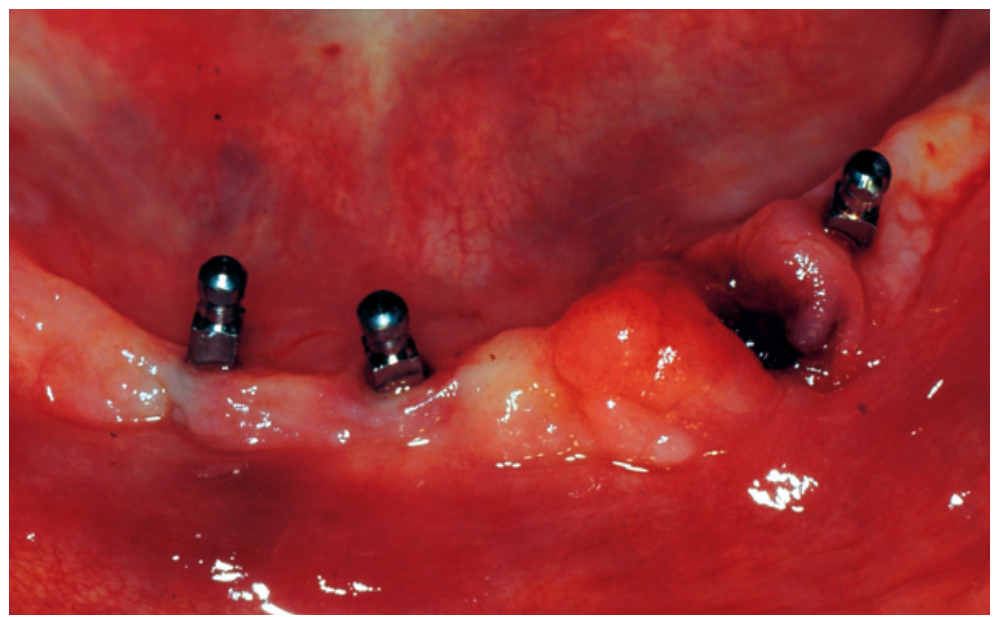

Figure 5 : Positionnement transmuqueux des mini-implants avec extraction de la 33.

Transmucous placement of mini implants with extraction of 33 . 
Un quatrième implant de $15 \mathrm{~mm}$ en position 43 n'a pas été positionné en raison de la sensibilité persistante du rameau incisif qui n'a pas pu être calmée par une nouvelle infiltration d'anesthésique. Les trois implants mis en place présentent une excellente stabilité primaire. Une orthopantomographie de contrôle est réalisée immédiatement après l'intervention. On procède à un évidement de l'intrados de la prothèse mandibulaire en regard de l'émergence de la tête des implants.
Le patient est revu la semaine suivante pour la mise en place des parties femelle des attachements sphériques par adjonction de résine autopolymérisable (Fig. 6). Les suites opératoires ont été simples et l'hyperesthésie du rameau incisif a disparu. Le patient est revenu une semaine plus tard pour un contrôle de l'équilibration occlusale. Un an après l'intervention, les mini-implants sont radiographiquement ostéointégrés et le patient est satisfait du résultat obtenu (Fig. 7 et 8). Les tests de percussion et de mobilité sont normaux.

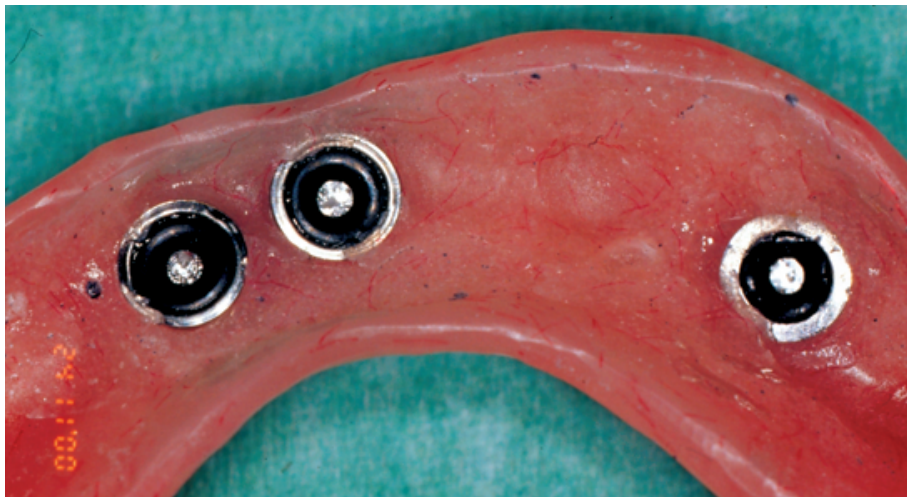

Figure 7 : Vue clinique 1 an après la pose. L'environnement tissulaire est parfait. Clinical view 1 year after placement. Tissue environment is perfect.

Figure 8 : Radiographie rétroalvéolaire 1 an après la pose. Les implants sont parfaitement ostéointégrés.

Radiograph 1 year after placement. The implants are perfectly osseointegrated.
Figure 6 : Intrados de la prothèse (gros plan) avec attachements en place.

Denture intrados with attachments (close up)

\section{buccale \\ chirurgie \\ buccale}

médecine

VOL. $9, \mathrm{~N}^{\circ} 1$ 2003

page 33

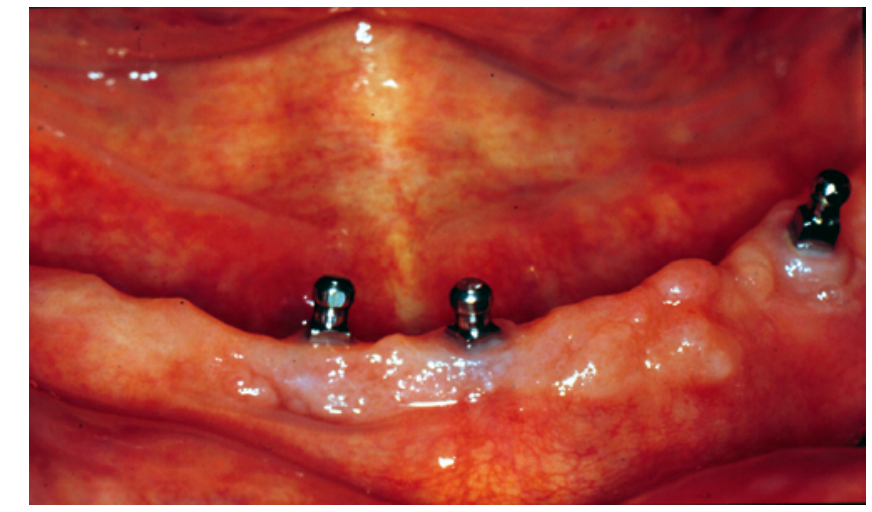

8

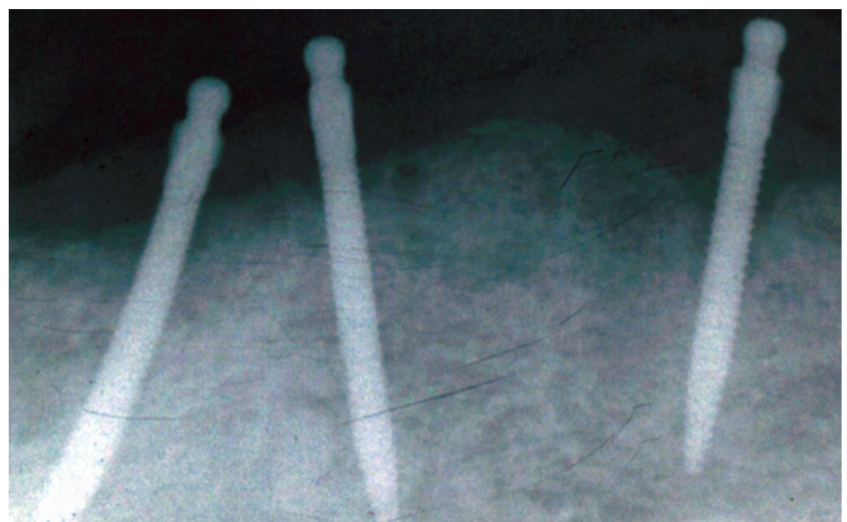


médecine

buccale

chirurgie

buccale

VOL. $9, \mathrm{~N}^{\circ} 1$ 2003

page 34

\section{DISCUSSION}

Les traitements présentés ont permis d'apporter aux patients une amélioration immédiate et durable de la stabilité et de la rétention de leur prothèse adjointe mandibulaire ainsi qu'un confort masticatoire accru. L'ensemble du traitement est de très courte durée (environ une semaine) et réalisé à moindre frais pour le patient. Dans le premier cas, la pose de miniimplants a évité la réalisation d'une greffe d'apposition osseuse en épaisseur, réduisant notablement à la fois le traumatisme post-opératoire subi par le patient, la durée globale et le coût du traitement. Un gain équivalent est apporté au second patient qui n'aurait pas pu avoir accès aux thérapeutiques implantaires classiques. Cependant, leur utilisation clinique présente pour l'instant un recul relativement faible (2 ans et demi). II convient d'observer avec vigilance leur évolution dans le temps même si la grande majorité (70 à $85 \%$ ) des échecs implantaires se produisent au cours des six premiers mois qui suivent la pose des implants et leur mise en charge [13-17]. La stabilité et la rétention de la prothèse sont satisfaisantes mais restent cependant inférieures à celles obtenues lors de la réalisation d'une barre implanto-portée conventionnelle.

Au travers des différents patients traités dans le service, la mise en charge immédiate de miniimplants non solidarisés entre eux est préconisée pour stabiliser à long terme une prothèse adjointe complète ou quasi complète. Pour I'instant, les limites sont fixées à l'arcade mandibulaire, entre les deux foramens mentonniers et chez un patient n'ayant pas de forces masticatrices trop importantes ni de parafonctions. Ce protocole peut permettre de résoudre sans chirurgie lourde (greffes autogènes d'épaississement) les problèmes des crêtes en lame de couteau dans la zone antérieure de la mandibule. C'est une alternative à l'implantologie classique chez la personne âgée, pour qui une intervention implantaire pourrait être mal supportée, mais aussi pour des patients dont les revenus sont modestes.

Au travers de la littérature, la mise en charge immédiate est à nouveau envisagée comme solution thérapeutique. Dans une étude, Schnitman et coll. [7] trouvent un taux de succès (tel que défini par Albrektsson et coll. [18]) de $85 \%$ à 10 ans pour les implants mis en charge immédiatement contre $100 \%$ pour ceux mis en nourrice. Les études menées par Randow et coll. [6], Tarnow et coll. [8] montrent des taux de succès comparables à condition de respecter des critères de répartition, de longueur et de sollicitation des implants. Randow et coll. [6] observent même une perte osseuse initiale inférieure sur les implants mis en charge immédiatement par rapport aux implants enfouis. D'autres études (Chiapasco et coll. [3], Jaffin et coll. [5]) sont plus difficilement exploitables en raison d'un trop grand nombre de patients perdus de vue ou de type d'implants très différents. Un cas d'implant unitaire placé au maxillaire et mis en charge immédiatement est même rapporté par Gomez et coll. [4] ouvrant la voie à de nouvelles perspectives. Les analyses histologiques chez l'homme effectuées par Piatelli et coll. [19] montrent des résultats compatibles avec un succès clinique. Ils attirent l'attention sur l'importance de la densité osseuse et de la stabilité primaire avec l'utilisation d'implants vissés et qui comportent un état de surface modifié. Les mini-implants répondent à ces caractéristiques : ils sont vissés et présentent un état de surface mordancé qui accroît la surface développée de l'implant. De plus, le forage est sous dimensionné par rapport à la taille de l'implant, ce qui leur permettent d'obtenir une stabilité primaire accrue. Balkin et coll. [11] confirment, par des études histologiques chez l'homme, la bonne ostéointégration des miniimplants après 4 et 5 mois de mise en charge immédiate.

La seule différence réside dans le diamètre de l'implant ; les études concernant le rôle du diamètre de l'implant sont partagées. On sait que la surface développée d'un implant dépend beaucoup de son diamètre, ainsi Ivanoff et coll. [20] montrent une augmentation statistiquement significative du couple de dévissage de l'implant corrélée avec celle du diamètre de l'implant. En revanche, Block et coll. [21] ont observé chez le chien que la résistance au 
retrait d'un implant ostéointégré dépendait de sa longueur mais pas de son diamètre. Sur des cadavres, Kido et coll. [22] ont trouvé pour la résistance au retrait une différence non significative de $15 \%$ entre les implants de petit et de gros diamètre. Dans la même étude, il y a une corrélation significative entre la densité osseuse et la résistance au retrait et ce quel que soit le diamètre de l'implant. Lors d'une étude portant sur les forces transmises aux implants lors de la phase de cicatrisation osseuse, Emmer et coll. [23] notent l'importance de l'adaptation des prothèses durant cette phase soit par évidement, soit par rebasage souple. La sollicitation des implants conditionne certainement le futur succès de l'ostéointégration, mais la frontière entre micromouvements et macromouvements est délicate à gérer cliniquement. Les attachements livrés avec les mini-implants présentent un certain degré de résilience et permettent une plus faible sollicitation de ceux-ci. De plus, leur stabilité primaire est accrue par leur forme autoforante et par l'utilisation d'un préforage sous dimensionné par rapport au diamètre de l'im- plant ; ceci contribue à limiter la sollicitation des mini-implants durant cette période.

Les mini-implants sont le plus souvent utilisés durant la période d'ostéointégration d'implants conventionnels [11,24] avant d'être déposés lorsque la réalisation prothétique implanto-portée finale est confectionnée [10,25]. Ceux-ci sont alors reliés par un artifice prothétique qui rigidifie l'ensemble et limite les forces transmises aux mini-implants. El Attar et coll. [9] préconisent I'utilisation de deux mini-implants non solidarisés entre eux pour stabiliser une prothèse adjointe durant la période d'ostéointégration d'implants conventionnels.

Compte tenu des limites et réserves émises dans cette étude, l'utilisation des mini-implants entre les foramens mentonniers pour la stabilisation immédiate et à long terme d'une prothèse adjointe mandibulaire semble représenter une alternative possible aux thérapeutiques implantaires traditionnelles et permet d'apporter aux patients une satisfaction et un réel bénéfice. médecine buccale chirurgie buccale

VOL. $9, \mathrm{~N}^{\circ} 1$ 2003

page 35

\section{RÉFÉRENCES}

1 - Bränemark PI, hansson BO, Adell R. Osseointegrated implants in the treatment of the edentulous jaw. Experience from a 10 years period. Scand J Plast Reconstr Surg 1977; 11 (suppl 16): 132.

2 - Davarpanah M, Hage G, Slama M, Tecucianu JF. Historique et évolution des concepts en implantologie. J Parodontol 1992; 11: 85-92.

3 - Chiapasco M, Gatti C, Rossi E, Haefliger W, MARKWALDER TH. Implant-retained mandibular overdentures with immediate loading: a retrospective multicenter study on 226 consecutive cases. Clin Oral Impl Res 1997; 8: 48-57.

4 - Gomez A, Lozada J, Caplanis N, Kleinman A. Immmediate loading of a single hydroxyapatite-coated threaded root form implant: a clinical report. J Oral Impl 1998; 24:159-66.

5 - JafFin RA, KUMAR A, BERMAN CL. Immediate loading of implants in partially and fully edentulous jaws: a series of 27 case reports. J Periodontol 2000; 71: 83-8.

6 - Randow K, ERicsson I, Nilner K, Peterssson A, Glantz $P O$. Immediate functional loading of Bränemark dental implants : a 18-month clinical follow-up study. Clin Oral Impl Res 1999; 10: 8-15.
7 - Schnitman PA, Wörle PS, Rubenstein Je, Da Silva JD, WANG NH. Ten year results for Bränemark implants immediately loaded with fixed protheses at implant placement. Int J Oral Maxillofac Impl 1997; 12: 495-503.

8 - TARNOW DP, EMTIAZ S, CLASSI A. Immediate loading of threaded implants at stage 1 surgery in edentulous arches: ten consecutive case reports with 1- to 5year data. Int J Oral Maxillofac Impl 1997; 12: 31924.

9 - El attar MS, El Shazly D, Osman S, El Domiati S, SALLOUM MG. Study of effect of using mini-transitional implants as temporary abutments in implant overdenture cases. Implant Dent 1999; 8: 152-8.

10 - FROUM S, EMtiAZ S, BLOOM MJ, SCOLNICK J, TARNOW DP. The use of transitional implants for immediate fixed temporary protheses in cases of implant restorations. Pract Periodont Aesthet Dent 1998; 10: 737-46.

11 - Balkin BE, Steflick DE, Naval F. Mini-dental implant insertion with the auto-advance technique for ongoing applications. J Oral Implantol 2001; 27: 32-7.

12 - VIGOLO P, GivanI A. Clinical evaluation of single-tooth mini-implant restorations: a five-year retrospective study. J Prosthet Dent 2000; 84: 50-4. 
13 - Bert M. Complications et échecs en implantologie (pp 151-8). Cdp, Paris, 1994.

14 - Esposito M, HiRsCH JM, LEKHOLM U, THOMSEN P. Biological factors contributing to failures of osseointegrated oral implants (I): success criteria and epidemiology. Eur J Oral Sci 1998; 106: 527-51.

15 - ESPOSITO M, HIRSCH JM, LEKHOLM U, THOMSEN P. Differential diagnosis and treatment strategies for biological complications and failing oral implants: a review of literature. Int J Oral Maxillofac Impl 1999; 14: 473-90.

16 - GoOdACRE CJ, Kan JYK, Rungcharassaeng K. Clinical complications of osseointegrated implants. J Prosthet Dent; 81: 537-52.

17 - TULASNE JF, RIACHI F. Complications des implants dentaires. J Parodontol 1991; 10: 219-25.

18 - Albrektsson T, Zarb GA, Worthington P, ReIKSSON AR. The long-term efficacy of currently used dental implants. A review and proposed criteria of success. Int J Oral Maxillofac Impl 1986; 1: 11-25.

19 - Piattelli A, Paolantonio M, Corigliano M, Scarano A. Immediate loading of titanium plasma-sprayed screw-shaped implants in man: a clinical and histolo-

médecine buccale chirurgie buccale

VOL. $9, \mathrm{~N}^{\circ} 1$ 2003

page 36
20 - IVAnoff CJ, Sennerby L, Johansson C, Rangert B, LEKHOLM U. Influence of implant diameters on the integration of screws implants. An experimental study in rabbits. Int J Oral Maxillofac Surg 1997; 26: 141-8.

21 - Block MS, Delgado A, Fontenot MG. The effect of diameter and length of hydroxyapatite-coated dental implants on ultimate pull-out force in dog alveolar bone. J Oral Maxillofac Surg 1990; 48: 174-8.

22 - Kido H, Schulz EE, Kumar A, Lozada J, Saha S. Implant diameter and bone density: effect on initial stability and pull-out resistance. J Oral Implantol 1997; 23: 163-9.

23 - EMMER TJ JR, EMmer TJ SR, VAIDYANATHAN J, VAIDYANATHAN TK. Measurement of submucosal forces transmitted to dental implants. J Oral Impl 1999; 25: 155-60.

24 - SendaX VI. Mini-implants as adjuncts for transitional prostheses [interview]. Dent Impl Update 1996; 7: 125.

25 - ZuBERY Y, BICHACHO N, MOSES O, TAL H. Immediate loading of modular transitional implants: a histologic and morphometric study in dogs. Int $\mathrm{J}$ Periodont Restorat Dent 1999; 19: 343-53. 